\title{
Petrology and chemistry of basal lherzolites above the metamorphic sole from Wadi Sarami central Oman ophiolite
}

\author{
Mohamed ZAKI KHEDR ${ }^{* * *}$, Shoji ARAI ${ }^{*}$ and Marie PYTHON ${ }^{* * *}$ \\ ${ }^{*}$ College of Science and Engineering, School of Natural System, Earth Science Course, \\ Kanazawa University, Kakuma, Kanazawa 920-1192, Japan \\ ** Department of Geology, Faculty of Science, Kafrelsheikh University, 33516, Egypt \\ ${ }^{* * *}$ Department of Natural History Sciences, Faculty of Science, Hokkaido University, \\ N10W8 Kita-ku, Sapporo 060-0810, Japan
}

\begin{abstract}
We studied basal lherzolites that are exposed along the metamorphic sole at the base of the central Oman ophiolite (Wadi Sarami). We recognized two types of lherzolites (Types I and II) based on field occurrences, textures, and mineral compositions. Type I lherzolites are massive and transition into harzburgites, whereas Type II lherzolites are strongly foliated with mylonitic to porphyroclastic textures. Type II lherzolites only crop out within a direct contact with the amphibolitic sole to few meters above this sole and are overlain and/or surrounded by Type I. The clinopyroxenes $[\mathrm{Mg} \#=\mathrm{Mg} /(\mathrm{Mg}+\mathrm{Fe})=0.89-0.94]$ of Type II lherzolites show higher contents of $\mathrm{Al}_{2} \mathrm{O}_{3}(4.5-7.3 \mathrm{wt} \%), \mathrm{Na}_{2} \mathrm{O}(0.5-1.2 \mathrm{wt} \%), \mathrm{Cr}_{2} \mathrm{O}_{3}(0.6-1.4 \mathrm{wt} \%)$, and $\mathrm{TiO}_{2}(0.2-0.4 \mathrm{wt} \%)$ than those of Type I lherzolites. Positive correlations among the $\mathrm{Al}_{2} \mathrm{O}_{3}, \mathrm{Na}_{2} \mathrm{O}$, and $\mathrm{TiO}_{2}$ contents of clinopyroxenes show a pronounced residual trend from Type I lherzolites to depleted harzburgite, giving rise to chemical heterogeneities at the base of the mantle section. Clinopyroxenes in lherzolites and harzburgites show compositional trends that are similar to those in abyssal peridotites from normal ridge segments. Olivines $\left(\mathrm{Fo}_{89.4}-\mathrm{Fo}_{91.5}\right)$ show a residual character of the Sarami peridotites. Primary spinels show a wide range of $\mathrm{Cr} \#[=\mathrm{Cr} /(\mathrm{Cr}+\mathrm{Al})$ from 0.04 to 0.53$]$ and low $\mathrm{Y}_{\mathrm{Fe}}\left[\mathrm{Fe}^{3+} /\left(\mathrm{Cr}+\mathrm{Al}+\mathrm{Fe}^{3+}\right),<0.046\right]$, similar to spinels in abyssal peridotites. The wide range of spinel $\mathrm{Cr} \#$ is a result of a wide range of partial-melting degrees, which are up to $10 \%$ for lherzolites and $\sim 10-25 \%$ for harzburgites. The Type II lherzolites, which occur near the paleo-fracture zone located to the east of Wadi Sarami, represent a remnant of asthenospheric materials trapped at the base of oceanic lithosphere mantle (Type I) during detachment and obduction. The Type I lherzolites experienced high-degree partial melting, resulting in the formation of harzburgites at the refractory end. The modal and compositional variations of Sarami pyroxenes and spinels indicate intrinsic mantle heterogeneity of Oman ophiolite formed as residues at an oceanic spreading center.
\end{abstract}

Keywords: Basal lherzolites, Abyssal peridotites, Clinopyroxene, Wadi Sarami, Central Oman ophiolite

\section{INTRODUCTION}

The Oman ophiolite is one of the best-preserved crustupper mantle sections of oceanic lithosphere in the world. Mantle sections in the Oman ophiolite consist mainly of harzburgites that have been formed by extensive partial melting at a fast spreading ridge (e.g., Boudier and Nicolas, 1985). Locally, minor dunites and lherzolites have been recognized in association with harzburgites. Basal lherzolites occur only at the base of the Oman ophiolite in a few places and have experienced strong deformation

doi:10.2465/jmps. 121026

M.Z. Khedr, khedrzm@yahoo.com Corresponding author and likely metasomatism, which are related to ophiolite obduction and emplacement. Few studies were devoted to the basal lherzolites, which were carefully described at only a few locations in the Fizh block in north Oman (Takazawa et al., 2003) and the Wadi Tayin in south Oman (Godard et al., 2000; Hanghøj et al., 2010). Godard et al. (2000) proposed that the clinopyroxene (Cpx)-bearing harzburgite (up to $5 \mathrm{vol} \%$ of $\mathrm{Cpx}$ ) identified in Wadi Tayin was formed by thermal erosion of the base of preexisting oceanic lithosphere by asthenospheric upwelling during oceanic rifting. However, Takazawa et al. (2003) divided the basal lherzolites into two types, I and II, from the Fizh block and considered their origin separately: the Fizh Type I lherzolites are residuals and are affected by 
melts enriched in light rare earth elements (LREE), whereas the Fizh Type II lherzolites were formed through the refertilization of refractory peridotite by freezing of trapped melt. There are many questions regarding the origin and petrogenetic relationships between the two types of lherzolites and harzburgites and their interaction with melts during the refertilization processes. To answer these questions, we conducted an extensive sampling of the basal peridotites of the Sarami and Wuqbah blocks along the Wadi Sarami area, which is an excellent area to study basal lherzolites and the peridotite-amphibolite interaction in the Oman ophiolite. The aim of this study is to clarify the origin and petrogenesis of the basal lherzolites from the Oman ophiolite and to understand how the mantle heterogeneity is related to a fast-rate of oceanic spreading. This is the first study to document two types of basal lherzolites from the Wadi Sarami central Oman ophiolite.

\section{FIELD AND PETROGRAPHICAL CHARACTERISTICS}

Wadi Sarami crosses from east to west the northern Hawasina window down to the base of the Wuqbah block. The studied area is divided by the Hawasina window into two main blocks (Sarami and Wuqbah). The Sarami block is the northernmost block of the central massifs of the Oman ophiolite (Nicolas et al., 2000) (Fig. 1a). The Semail ophiolitic nappe, which is composed of harzburgites and dunites with minor lherzolites, gabbros, and sheeted dyke complex, is thrusted over the metamorphic soles (mainly amphibolites) and the Hawasina nappe (carbonate, clastic, and pelagic sediments) (Ministry of Petroleum and Minerals, 1992) around Wadi Sarami (Fig. 1). We collected over 200 samples of basal peridotites along the Wadi Sarami from the base of the Sarami and Wuqbah blocks from three locations (Al-Khabt, Al-Baks, and AlQala, see Fig. 1b) to examine the lithological and chemi-

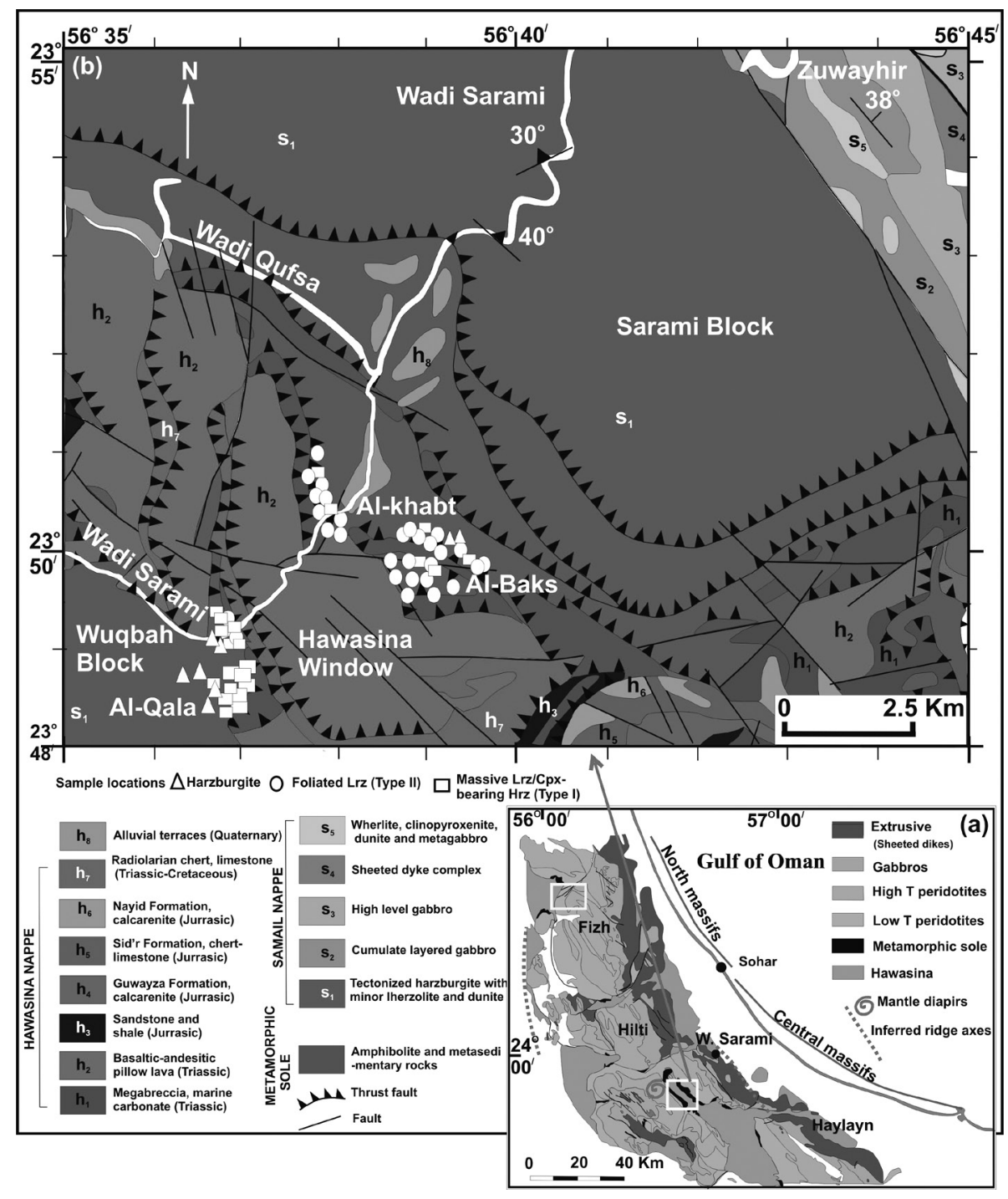

Figure 1. Geological map of basal peridotites from the Wadi Sarami central Oman ophiolite. (a) A part of main ridge-related structural map of the Oman ophiolite showing the Wadi Fizh area (yellow rectangular) studied by Takazawa et al. (2003), inferred ridge axes, and the central and north ophiolite massifs (Nicolas et al., 2000). (b) Geological map of the Wadi Sarami showing the locations of basal peridotite samples from the base of the Sarami and Wuqbah blocks (modified from the map of the Ministry of Petroleum and Minerals, 1992). 
cal changes from the lherzolites to the harzburgites (Fig. 2a). We recognized two types of basal lherzolites, which are almost equivalent to the Type I and II lherzolites described by Takazawa et al. (2003) (Fig. 2). Type I lherzolites are massive (Figs. 2a and 2b) and crop out at various levels, from the direct contact with the sole and/or Type II lherzolites to few hundreds meters stratigraphically above the metamorphic sole in both the Sarami and Wuqbah blocks (Fig. 1b). They are homogenous masses and are less serpentinized (no pyroxene pseudomorphs) (Figs. 2a and $2 \mathrm{~b}$ ) relative to Type II lherzolites. The contact between Type I and Type II lherzolites is not directly visible. The transition from massive lherzolites (Type I) to harzburgites at Al-Qala village appears to be gradual. The Type I lherzolites have been upward changed to harzburgites (Fig. 2a). Type II lherzolites show a strong foliation parallel to the amphibolite foliation of NW-SE trend (Fig. 2c), and they are located at the base of the Sarami block (Fig. 1b). The Type II lherzolites look like heterogeneous masses because of the occurrence of the sole pieces (e.g., amphibolites, metasediment) mixed with the altered or serpentinized surfaces of some lherzolite parts. They extend from the base directly on the metamorphic sole up to few meters (sometimes up to $\sim 150 \mathrm{~m}$ ), and they are overlain and/or surrounded by Type I lherzolites. Type II lherzolites are highly serpentinized and rich in pyroxene pseudomorphs (serpentines: bastites) (Fig. 2e), and they exhibit sharp contacts with amphibolites (Fig. 2d). They also suffer from low-temperature shearing and mylonitic deformation at the base of the mantle section above the sole. The mylonitic deformation could be related to a detachment fault during tectonic exhumation or localized ductile shear zones in the lithospheric mantle (e.g., Michibayashi et al., 2009). In some places (Al-Baks and AlKhabt villages), Type II lherzolites, cropping out as huge tectonic masses or ridges, are surrounded by amphibolites, and look like trapped blocks in Type I lherzolites above the metamorphic sole (Fig. 2c). The failing ridge or paleo-fracture zone (Nicolas et al., 2000) is located to the east of Wadi Sarami close to the location of the Type II exposure (Fig. 1a), similar to the distribution in the northern Fizh block (Nicolas et al., 2000; Takazawa et al., 2003). Type II lherzolites occur near the segment end, and they are absent in Al-Qala village (Wuqbah block), which is far from the segment end (Nicolas et al., 2000) (Fig. 1a). All basal lherzolites in Wadi Sarami are free of any evidence of later magmatic activities or melt refertilization, i.e., dykes, veins (dunite bands or dunite veins), sills, and melt channels.

Type I lherzolites are weakly to moderately serpentinized (7-53 vol\%) with subordinate amounts of pargasitic hornblende, tremolite, and magnetite; they consist of the following primary minerals: olivine (4.1-66.5 vol\%), orthopyroxene (Opx) (14.0-27.2 vol\%), Cpx (4.0-14.1 vol\%), and spinel (0.4-1.1 vol\%) (Figs. 3a and 3b). The modal abundance is based on point counting (2000 counts per $2.5 \times 4.5 \mathrm{~cm})$. Type I lherzolites exhibit porphyroclastic textures (Fig. 3a). Some coarse prismatic crystals of Cpx (2.0-2.4 mm) contain exsolution lamellae from Alspinel (Fig. 3b). Olivine occurs as small rounded interlocking grains around Opx and Cpx porphyroclasts (Fig. $3 a)$. Some Opx porphyroclasts ( $4 \mathrm{~mm}$ in width) are surrounded by corona from fibrous tremolites, which are alteration products of Opx. Type II lherzolites are moderately to highly serpentinized (37-75 vol\%) with subordinate pargasitic hornblende, chlorite, and magnetite; they are composed of the following primary minerals: olivine (0.0-17.9 vol\%), Opx (7.2-26.5 vol\%), Cpx (4.7-10.2 vol\%), and spinel (0.75-1.5 vol\%) (Figs. 3c-3f). They show mainly mylonitic to porphyroclastic textures (Figs. $3 \mathrm{c}$ and $3 \mathrm{~d})$. Cpx shows various morphologies, such as coarse euhedral $(2.0-2.5 \mathrm{~mm})$ to subhedral laminated crystals, neoblasts, and fine matrix grains (Figs. 3c and 3d). The Cpx crystals are aligned in a definite trend, forming mineral lineations (Fig. 3e). They are sometimes partly replaced or pitted by hornblende minerals (Fig. 3f). Opx occurs as large prismatic crystals (up to $4.8 \mathrm{~mm}$ in width) surrounded by a groundmass consisting of fine grains of Opx, Cpx, and olivine. The fine-grained matrix warps around Opx porphyroclasts to form a mylonitic texture (Fig. 3c). Most Opx porphyroclasts, showing kink bands and bending because of emplacement stresses, contain blebs or exsolution lamellae of Cpx. Primary spinels are vermicular in shape and orange to red colored in thin sections, and they occur interstitially between pyroxene grains. The olivine-rich matrix is equigranular. The serpentines in the matrix are mainly lizardite and chrysotile. Harzburgites are only weakly serpentinized ( $<15$ vol\%). They consist of olivine (71-85 vol\%), Cpx (1.7-3 vol\%), Opx (11-13.7 vol\%), and spinel (0.3-0.5 vol\%), and they are characterized by protogranular textures. Primary spinels, which are red to black in color in transmitted light in thin sections, occur as subhedral to anhedral grains and include inclusions of olivine, Opx, and Cpx. Cpx, occurring as subhedral to euhedral crystals, includes exsolved lamellae of Opx.

\section{MINERAL CHEMISTRY}

An electron microprobe (JXA-8800, JEOL) at Kanazawa University, Japan, was used to determine the major-element compositions of silicate minerals and spinels in basal peridotites from the Wadi Sarami central Oman ophiolite (Table 1). The accelerating voltage, beam current, and 

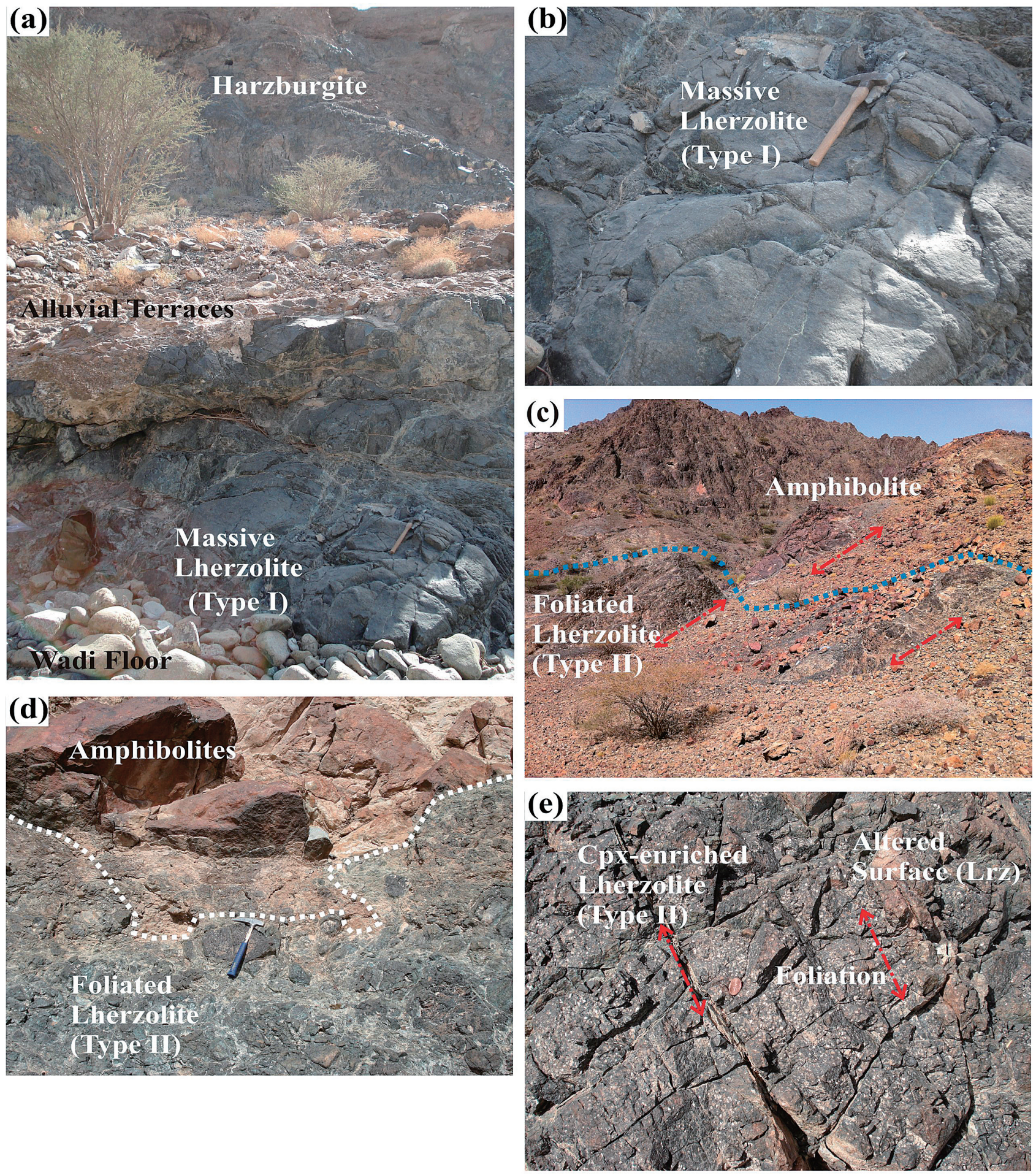

Figure 2. Field photographs of the Sarami basal peridotites from the central Oman ophiolite. (a) Massive lherzolites (Type I) in the base of peridotites, overlain by harzburgites. The Type I lherzolites, covered by surficial thin alluvial terraces, have been changed to harzburgites as huge masses (small scale: $<100 \mathrm{~m}$ ). (b) Magnified view of massive lherzolites (Type I) without structure or foliation. Type I lherzolites are fresh (without pyroxene pseudomorphs) homogenous boulders compared to Type II lherzolites. (c) Type II lherzolites showing a strong foliation parallel to amphibolite foliation. (d) Sharp contact between amphibolites (the sole) and foliated lherzolites (Type II). (e) Foliated lherzolites (Type II) enriched with pyroxenes and pyroxene pseudomorph (serpentines: bastites). The Type II lherzolites look like heterogeneous masses because of the occurrence of the sole pieces (e.g., amphibolites, metasediment) mixed with altered or serpentinized surfaces of some lherzolite (Lrz) components. 

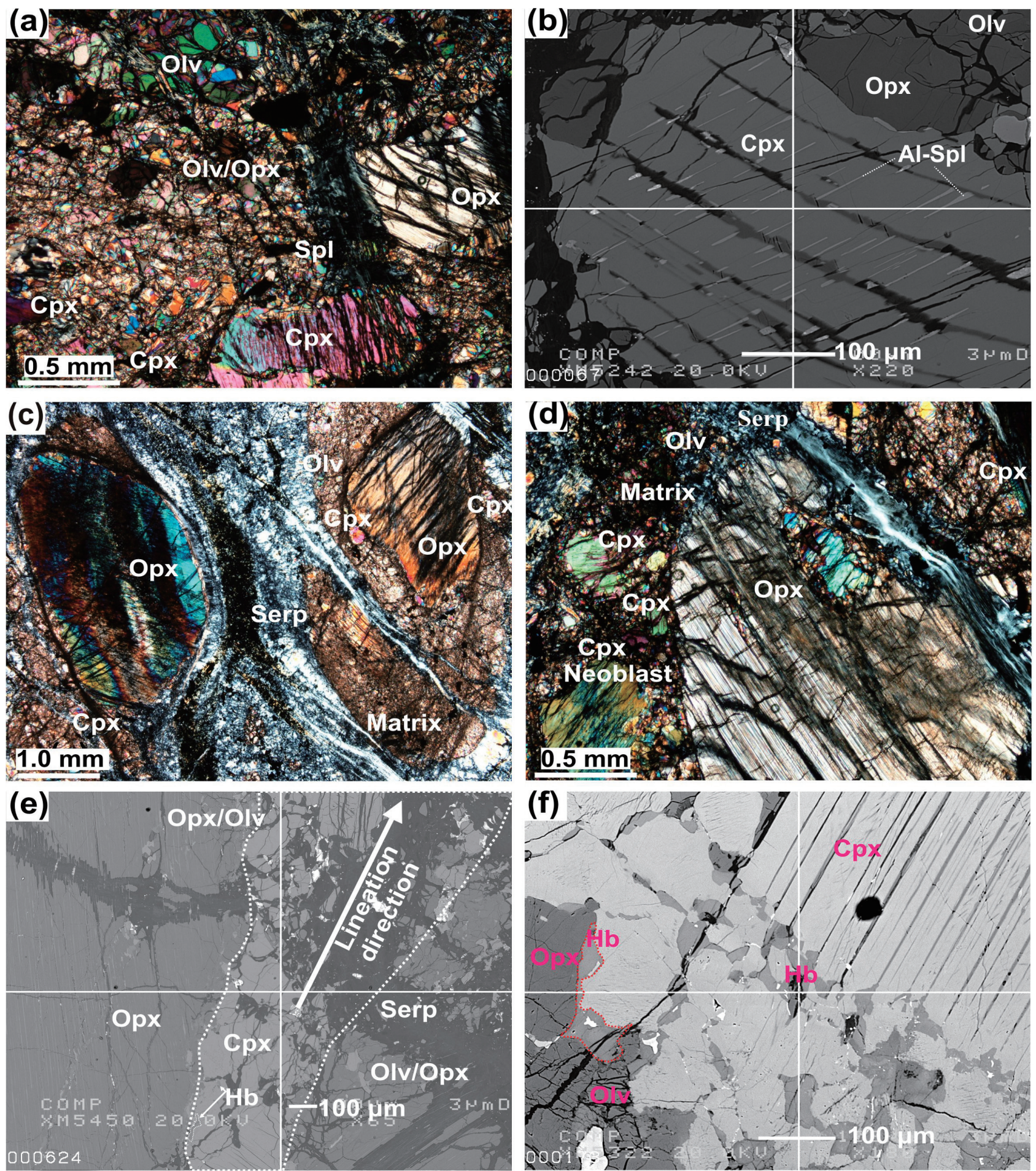

Figure 3. Photomicrographs of basal peridotites from Wadi Sarami central Oman ophiolite. Crossed-polarized light was used except for subplots (b), (e), and (f) (backscattered electron image, BSE). (a) Equigranular olivine around Opx and Cpx porphyroclasts in massive Type I lherzolites (Tv. 128), showing porphyroclastic textures. (b) Prismatic crystals of Cpx containing exsolution lamellae of Al-spinel, reflecting high $\mathrm{Al}$ in the lherzolitic bulk composition. (c) A fine-grained matrix (Opx $+\mathrm{Cpx}+$ olivine) warped around Opx porphyroclasts in foliated Type II lherzolite (Tv. 45), showing mylonitic textures. (d) Opx porphyroclasts and Cpx neoblasts embedded in fine-grained olivine + Opx + Cpx (Tv. 40), suggesting porphyroclastic textures. (e) Cpx crystals aligned in a definite trend with Opx, olivine, and Hb, forming mineral lineations in foliated lherzolites (Tv. 40). (f) Cpx crystals occasionally replaced or pitted by hornblende to form replasive or reaction textures in foliated lherzolites (G. 27). Abbreviations; Opx, orthopyroxene; Cpx, clinopyroxene; Olv, olivine; Spl, primary spinel; Serp, serpentines; Hb, hornblende. 
Table 1. Representative microprobe analyses of silicate minerals and spinels in Sarami

\begin{tabular}{|c|c|c|c|c|c|c|c|c|c|c|c|c|c|}
\hline \multirow{3}{*}{$\begin{array}{l}\text { Rock } \\
\text { Sample N. } \\
\text { Minearls } \\
\end{array}$} & \multicolumn{13}{|c|}{ Type I lherzolites } \\
\hline & \multicolumn{4}{|c|}{ Tv.56 } & \multicolumn{5}{|c|}{ Tv.127 } & \multicolumn{4}{|c|}{ G.33 } \\
\hline & Olv & $\mathrm{Cpx}$ & Opx & Spl & Olv & $\mathrm{Cpx}$ & Opx & Par.Hb & Spl & Olv & $\mathrm{Cpx}$ & Opx & Spl \\
\hline $\mathrm{SiO}_{2}$ & 40.48 & 52.36 & 55.32 & 0.00 & 39.53 & 51.45 & 57.77 & 46.50 & 0.02 & 40.73 & 53.28 & 55.13 & 0.01 \\
\hline $\mathrm{TiO}_{2}$ & 0.02 & 0.24 & 0.08 & 0.05 & 0.00 & 0.25 & 0.02 & 0.55 & 0.02 & 0.01 & 0.16 & 0.03 & 0.05 \\
\hline $\mathrm{Al}_{2} \mathrm{O}_{3}$ & 0.00 & 4.44 & 4.57 & 54.40 & 0.00 & 4.48 & 2.65 & 12.97 & 46.86 & 0.00 & 3.31 & 4.57 & 45.26 \\
\hline $\mathrm{Cr}_{2} \mathrm{O}_{3}$ & 0.00 & 0.93 & 0.62 & 13.90 & 0.00 & 0.85 & 0.25 & 0.65 & 20.77 & 0.00 & 0.69 & 0.83 & 22.17 \\
\hline $\mathrm{FeO}^{*}$ & 9.56 & 2.11 & 6.03 & 13.73 & 10.41 & 2.22 & 6.60 & 3.75 & 14.36 & 9.10 & 2.03 & 6.10 & 14.26 \\
\hline $\mathrm{MnO}$ & 0.15 & 0.06 & 0.15 & 0.11 & 0.10 & 0.08 & 0.19 & 0.02 & 0.13 & 0.11 & 0.06 & 0.12 & 0.15 \\
\hline $\mathrm{MgO}$ & 50.16 & 16.35 & 32.69 & 18.28 & 49.26 & 16.37 & 35.01 & 18.37 & 17.51 & 50.08 & 16.47 & 33.13 & 17.80 \\
\hline $\mathrm{CaO}$ & 0.02 & 23.70 & 1.65 & 0.00 & 0.01 & 23.98 & 0.54 & 12.57 & 0.02 & 0.01 & 23.87 & 0.66 & 0.01 \\
\hline $\mathrm{Na}_{2} \mathrm{O}$ & 0.00 & 0.35 & 0.03 & 0.01 & 0.00 & 0.24 & 0.01 & 2.09 & 0.00 & 0.00 & 0.25 & 0.00 & 0.01 \\
\hline $\mathrm{K}_{2} \mathrm{O}$ & 0.00 & 0.00 & 0.00 & 0.00 & 0.00 & 0.00 & 0.00 & 0.00 & 0.00 & 0.00 & 0.00 & 0.00 & 0.00 \\
\hline $\mathrm{NiO}$ & 0.36 & 0.03 & 0.08 & 0.29 & 0.41 & 0.04 & 0.08 & 0.09 & 0.32 & 0.38 & 0.06 & 0.06 & 0.22 \\
\hline Total & 100.7 & 100.6 & 101.2 & 100.8 & 99.7 & 100.0 & 103.1 & 97.6 & 100.0 & 100.4 & 100.2 & 100.6 & 99.9 \\
\hline${ }^{\mathrm{a}} \mathrm{Mg} \#$ & 0.90 & 0.93 & 0.91 & 0.72 & 0.89 & 0.93 & 0.90 & 0.90 & 0.71 & 0.91 & 0.94 & 0.91 & 0.73 \\
\hline${ }^{\mathrm{b}} \mathrm{Fe \#}$ & 0.10 & 0.07 & 0.09 & 0.28 & 0.11 & 0.07 & 0.10 & 0.10 & 0.29 & 0.91 & 0.06 & 0.09 & 0.27 \\
\hline${ }^{\mathrm{c}} \mathrm{Cr} \#$ & & 0.12 & 0.08 & 0.15 & & 0.11 & 0.06 & 0.03 & 0.23 & & 0.12 & 0.11 & 0.25 \\
\hline Wo & & 49.23 & 3.18 & & & 49.39 & 0.99 & & & & 49.29 & 1.28 & \\
\hline En & & 47.25 & 87.54 & & & 46.91 & 89.30 & & & & 47.33 & 89.30 & \\
\hline $\mathrm{Y}_{\mathrm{Al}}$ & & & & 0.84 & & & & & 0.75 & & & & 0.73 \\
\hline $\mathrm{Y}_{\mathrm{Cr}}$ & & & & 0.14 & & & & & 0.22 & & & & 0.24 \\
\hline $\mathrm{Y}_{\mathrm{Fe} 3+}$ & & & & 0.01 & & & & & 0.02 & & & & 0.03 \\
\hline
\end{tabular}

\begin{tabular}{|c|c|c|c|c|c|c|c|c|c|c|c|c|c|c|}
\hline Rock & & & & & & & ype II ll & nerzolite & & & & & & \\
\hline Sample N. & & $\mathrm{G}$. & & & & G. & & & N.2 & 77 & & Tv. & 121 & \\
\hline Minearls & Olv & Cpx & Opx & Spl & $\mathrm{Olv}$ & Cpx & Opx & Spl & $\mathrm{Cpx}$ & Opx & Olv & $\mathrm{Cpx}$ & Opx & Spl \\
\hline$\overline{\mathrm{SiO}_{2}}$ & 41.09 & 51.05 & 54.27 & 0.00 & 40.67 & 51.56 & 53.51 & 0.01 & 52.81 & 54.53 & 40.60 & 52.17 & 55.21 & 0.00 \\
\hline $\mathrm{TiO}_{2}$ & 0.00 & 0.40 & 0.12 & 0.05 & 0.01 & 0.29 & 0.06 & 0.03 & 0.29 & 0.11 & 0.00 & 0.30 & 0.06 & 0.01 \\
\hline $\mathrm{Al}_{2} \mathrm{O}_{3}$ & 0.00 & 6.90 & 5.88 & 58.45 & 0.00 & 6.37 & 6.13 & 56.30 & 5.04 & 5.60 & 0.00 & 6.37 & 5.26 & 56.98 \\
\hline $\mathrm{Cr}_{2} \mathrm{O}_{3}$ & 0.00 & 0.89 & 0.57 & 10.94 & 0.00 & 0.90 & 0.64 & 11.83 & 0.94 & 0.92 & 0.00 & 0.97 & 0.52 & 10.81 \\
\hline $\mathrm{FeO}^{*}$ & 9.63 & 2.50 & 6.12 & 11.80 & 9.59 & 2.44 & 6.03 & 11.62 & 2.11 & 5.64 & 10.21 & 2.26 & 6.22 & 11.86 \\
\hline $\mathrm{MnO}$ & 0.15 & 0.07 & 0.12 & 0.11 & 0.14 & 0.09 & 0.17 & 0.13 & 0.07 & 0.14 & 0.15 & 0.04 & 0.15 & 0.11 \\
\hline $\mathrm{MgO}$ & 49.62 & 15.00 & 31.59 & 20.16 & 49.47 & 14.72 & 31.70 & 19.45 & 15.91 & 31.37 & 49.52 & 15.51 & 32.51 & 19.07 \\
\hline $\mathrm{CaO}$ & 0.01 & 21.50 & 1.28 & 0.00 & 0.02 & 22.47 & 0.71 & 0.00 & 23.57 & 2.34 & 0.02 & 22.77 & 1.34 & 0.01 \\
\hline $\mathrm{Na}_{2} \mathrm{O}$ & 0.01 & 1.04 & 0.08 & 0.00 & 0.00 & 1.12 & 0.03 & 0.01 & 0.68 & 0.11 & 0.00 & 0.93 & 0.03 & 0.00 \\
\hline $\mathrm{K}_{2} \mathrm{O}$ & 0.00 & 0.00 & 0.01 & 0.00 & 0.00 & 0.00 & 0.01 & 0.00 & 0.00 & 0.01 & 0.00 & 0.00 & 0.00 & 0.00 \\
\hline $\mathrm{NiO}$ & 0.35 & 0.04 & 0.09 & 0.31 & 0.36 & 0.03 & 0.10 & 0.32 & 0.05 & 0.07 & 0.39 & 0.04 & 0.09 & 0.33 \\
\hline Total & 100.9 & 99.4 & 100.1 & 101.8 & 100.3 & 100.0 & 99.1 & 99.7 & 101.5 & 100.8 & 100.9 & 101.3 & 101.4 & 99.2 \\
\hline${ }^{\mathrm{a}} \mathrm{Mg \#}$ & 0.90 & 0.91 & 0.90 & 0.77 & 0.90 & 0.91 & 0.90 & 0.76 & 0.93 & 0.91 & 0.90 & 0.92 & 0.90 & 0.75 \\
\hline${ }^{b} \mathrm{Fe \#}$ & 0.10 & 0.09 & 0.10 & 0.23 & 0.10 & 0.09 & 0.10 & 0.24 & 0.07 & 0.09 & 0.10 & 0.08 & 0.10 & 0.25 \\
\hline${ }^{\mathrm{c}} \mathrm{Cr} \#$ & & 0.08 & 0.06 & 0.11 & & 0.09 & 0.07 & 0.12 & 0.11 & 0.10 & & 0.09 & 0.06 & 0.11 \\
\hline Wo & & 48.45 & 2.55 & & & 50.00 & 1.43 & & 49.72 & 4.63 & & 49.34 & 2.60 & \\
\hline En & & 47.02 & 87.72 & & & 45.59 & 88.82 & & 46.70 & 86.44 & & 46.77 & 87.75 & \\
\hline $\mathrm{Y}_{\mathrm{Al}}$ & & & & 0.88 & & & & 0.87 & & & & & & 0.88 \\
\hline $\mathrm{Y}_{\mathrm{Cr}}$ & & & & 0.11 & & & & 0.12 & & & & & & 0.11 \\
\hline $\mathrm{Y}_{\mathrm{Fe} 3+}$ & & & & 0.01 & & & & 0.01 & & & & & & 0.01 \\
\hline
\end{tabular}

${ }^{*}$ Total iron as $\mathrm{FeO} .{ }^{\mathrm{a}} \mathrm{Mg} \#=\mathrm{Mg} /\left(\mathrm{Mg}+\mathrm{Fe}^{2+}\right)$ of silicates and $\mathrm{Fe}^{2+}$ as total iron but in case of spinels $\mathrm{Fe}^{2+}$ as a ferrous iron.

${ }^{\mathrm{b}} \mathrm{Fe} \#=\mathrm{Fe}^{2+} /\left(\mathrm{Mg}+\mathrm{Fe}^{2+}\right) \cdot{ }^{\mathrm{c}} \mathrm{Cr} \#=\mathrm{Cr} /(\mathrm{Cr}+\mathrm{Al})$.

$\mathrm{Y}_{\mathrm{Cr}}, \mathrm{Y}_{\mathrm{Al}}$, and $\mathrm{Y}_{\mathrm{Fe}}$ are the atomic ratios of $\mathrm{Cr}, \mathrm{Al}$, and $\mathrm{Fe}^{3+}$, respectively, to the trivalent cations, $\left(\mathrm{Cr}+\mathrm{Al}+\mathrm{Fe}^{3+}\right)$.

Ferrous and ferric iron contents of spinels were calculated assuming spinel stoichiometry.

Olv, olivine; Cpx, clinopyroxene; $\mathrm{Cpx}-\mathrm{c}$, core of clinopyroxene; Cpx-r, rim of clinopyroxene; Opx, orthopyroxene; Spl, spinel; Par.Hb, pargasitic hornblende. 
basal peridotites

\begin{tabular}{|c|c|c|c|c|c|c|c|}
\hline \multirow{3}{*}{$\begin{array}{l}\text { Rock } \\
\text { Sample N. } \\
\text { Minearls }\end{array}$} & \multicolumn{7}{|c|}{ Type I lherzolites } \\
\hline & \multicolumn{4}{|c|}{ G.40 } & \multicolumn{3}{|c|}{ Tv. 45} \\
\hline & Olv & Cpx & Par.Hb & $\mathrm{Spl}$ & $\mathrm{Cpx}$ & Opx & $\mathrm{Cr}-\mathrm{Spl}$ \\
\hline $\mathrm{SiO}_{2}$ & 40.41 & 53.42 & 44.67 & 0.00 & 53.01 & 55.01 & 0.00 \\
\hline $\mathrm{TiO}_{2}$ & 0.00 & 0.18 & 0.58 & 0.01 & 0.37 & 0.11 & 0.02 \\
\hline $\mathrm{Al}_{2} \mathrm{O}_{3}$ & 0.00 & 3.99 & 13.59 & 51.66 & 5.19 & 4.69 & 53.10 \\
\hline $\mathrm{Cr}_{2} \mathrm{O}_{3}$ & 0.00 & 0.86 & 1.39 & 16.91 & 0.89 & 0.60 & 16.23 \\
\hline $\mathrm{FeO} *$ & 8.41 & 1.88 & 3.09 & 12.01 & 2.11 & 5.74 & 13.02 \\
\hline $\mathrm{MnO}$ & 0.11 & 0.05 & 0.07 & 0.12 & 0.05 & 0.14 & 0.14 \\
\hline $\mathrm{MgO}$ & 50.86 & 16.99 & 18.57 & 18.42 & 16.75 & 32.12 & 18.90 \\
\hline $\mathrm{CaO}$ & 0.01 & 23.99 & 12.71 & 0.00 & 23.23 & 2.00 & 0.00 \\
\hline $\mathrm{Na}_{2} \mathrm{O}$ & 0.00 & 0.41 & 2.79 & 0.00 & 0.38 & 0.01 & 0.01 \\
\hline $\mathrm{K}_{2} \mathrm{O}$ & 0.01 & 0.00 & 0.00 & 0.00 & 0.00 & 0.00 & 0.00 \\
\hline $\mathrm{NiO}$ & 0.36 & 0.03 & 0.11 & 0.29 & 0.02 & 0.09 & 0.28 \\
\hline Total & 100.2 & 101.8 & 97.6 & 99.4 & 102.0 & 100.5 & 101.7 \\
\hline${ }^{\mathrm{a}} \mathrm{Mg \#}$ & 0.92 & 0.94 & 0.91 & 0.74 & 0.93 & 0.91 & 0.74 \\
\hline${ }^{\mathrm{b}} \mathrm{Fe} \#$ & 0.92 & 0.06 & 0.09 & 0.26 & 0.07 & 0.09 & 0.26 \\
\hline${ }^{\mathrm{c}} \mathrm{Cr} \#$ & & 0.13 & 0.06 & 0.18 & 0.10 & 0.08 & 0.17 \\
\hline Wo & & 48.82 & & & 48.17 & 3.90 & \\
\hline En & & 48.12 & & & 48.33 & 87.15 & \\
\hline $\mathrm{Y}_{\mathrm{Al}}$ & & & & 0.82 & & & 0.82 \\
\hline $\mathrm{Y}_{\mathrm{Cr}}$ & & & & 0.18 & & & 0.17 \\
\hline $\mathrm{Y}_{\mathrm{Fe} 3+}$ & & & & 0.00 & & & 0.01 \\
\hline \multicolumn{8}{|c|}{ Type II lherzolites } \\
\hline Sample N. & \multicolumn{4}{|c|}{ Tv.46 } & \multicolumn{3}{|c|}{ Tv.117 } \\
\hline Minearls & Olv & Cpx & Opx & Spl & Cpx & Opx & Spl \\
\hline$\overline{\mathrm{SiO}_{2}}$ & 41.77 & 52.19 & 54.62 & 0.00 & 53.61 & 55.61 & 0.01 \\
\hline $\mathrm{TiO}_{2}$ & 0.00 & 0.41 & 0.09 & 0.04 & 0.24 & 0.06 & 0.00 \\
\hline $\mathrm{Al}_{2} \mathrm{O}_{3}$ & 0.00 & 6.51 & 5.45 & 55.50 & 6.20 & 5.73 & 58.36 \\
\hline $\mathrm{Cr}_{2} \mathrm{O}_{3}$ & 0.00 & 1.05 & 0.61 & 13.14 & 1.11 & 0.68 & 11.60 \\
\hline $\mathrm{FeO}^{*}$ & 9.40 & 2.03 & 6.15 & 12.52 & 1.97 & 5.95 & 10.49 \\
\hline $\mathrm{MnO}$ & 0.13 & 0.08 & 0.13 & 0.14 & 0.06 & 0.14 & 0.12 \\
\hline $\mathrm{MgO}$ & 51.57 & 15.96 & 32.71 & 19.54 & 15.46 & 33.16 & 20.27 \\
\hline $\mathrm{CaO}$ & 0.01 & 22.57 & 0.91 & 0.00 & 22.76 & 0.50 & 0.00 \\
\hline $\mathrm{Na}_{2} \mathrm{O}$ & 0.00 & 1.07 & 0.04 & 0.01 & 1.04 & 0.02 & 0.02 \\
\hline $\mathrm{K}_{2} \mathrm{O}$ & 0.00 & 0.00 & 0.00 & 0.00 & 0.01 & 0.01 & 0.00 \\
\hline $\mathrm{NiO}$ & 0.37 & 0.04 & 0.09 & 0.30 & 0.00 & 0.08 & 0.30 \\
\hline Total & 103 & 101.9 & 100.8 & 101.2 & 102.4 & 101.9 & 101.2 \\
\hline${ }^{\mathrm{a}} \mathrm{Mg \#}$ & 0.91 & 0.93 & 0.90 & 0.76 & 0.93 & 0.91 & 0.78 \\
\hline${ }^{\mathrm{b}} \mathrm{Fe} \#$ & 0.09 & 0.07 & 0.10 & 0.24 & 0.07 & 0.09 & 0.22 \\
\hline${ }^{\mathrm{c}} \mathrm{Cr} \#$ & & 0.10 & 0.07 & 0.14 & 0.11 & 0.07 & 0.12 \\
\hline Wo & & 48.62 & 1.77 & & 49.65 & 0.97 & \\
\hline En & & 47.83 & 88.68 & & 46.90 & 89.78 & \\
\hline $\mathrm{Y}_{\mathrm{Al}}$ & & & & 0.85 & & & 0.88 \\
\hline $\mathrm{Y}_{\mathrm{Cr}}$ & & & & 0.13 & & & 0.12 \\
\hline $\mathrm{Y}_{\mathrm{Fe} 3+}$ & & & & 0.02 & & & 0.00 \\
\hline
\end{tabular}

beam diameter for the analyses were $20 \mathrm{kV}, 20 \mathrm{nA}$, and 3 $\mu \mathrm{m}$, respectively. The $\mathrm{Mg}$-number $(\mathrm{Mg} \#)$ is the atomic ratio of $\mathrm{Mg} /\left(\mathrm{Mg}+\mathrm{Fe}^{2+}\right)$, assuming that all $\mathrm{Fe}$ was ferrous for silicates. The $\mathrm{Cr}$-number $(\mathrm{Cr} \#)$ is the atomic ratio of $\mathrm{Cr} /(\mathrm{Cr}+\mathrm{Al})$. Ferrous and ferric iron contents of chromian spinel were estimated assuming spinel stoichiometry. $\mathrm{Y}_{\mathrm{Cr}}$, $\mathrm{Y}_{\mathrm{Al}}$, and $\mathrm{Y}_{\mathrm{Fe}}$ are the atomic ratios of the spinel $\mathrm{Cr}, \mathrm{Al}$, and $\mathrm{Fe}^{3+}$, respectively, to the trivalent cations $\left(\mathrm{Cr}+\mathrm{Al}+\mathrm{Fe}^{3+}\right)$. Clinopyroxenes (Cpxs) in both types (I and II) of lherzolites are diopside. $\mathrm{Cpxs}(\mathrm{Mg} \#=0.89-0.94)$ in Type II lherzolites show higher contents of $\mathrm{Al}_{2} \mathrm{O}_{3}$ (4.5-7.3 wt\%), $\mathrm{Na}_{2} \mathrm{O}$ (0.5-1.2 wt\%), $\mathrm{Cr}_{2} \mathrm{O}_{3}(0.6-1.4 \mathrm{wt} \%)$, and $\mathrm{TiO}_{2}(0.2-0.4 \mathrm{wt} \%)$ than those of Type I (Mg\#, $\leq 0.95$; $\mathrm{Al}_{2} \mathrm{O}_{3}$, mainly $<5.5 \mathrm{wt} \% ; \mathrm{Na}_{2} \mathrm{O},<0.6 \mathrm{wt} \% ; \mathrm{Cr}_{2} \mathrm{O}_{3},<1.2$ wt $\%$; and $\mathrm{TiO}_{2},<0.37 \mathrm{wt} \%$ ) (Table 1 and Fig. 4). The Cpxs show intra-grain chemical homogeneity (except for $\mathrm{Al}_{2} \mathrm{O}_{3}$ and $\mathrm{Na}_{2} \mathrm{O}$ ), but show inter-grain chemical heterogeneity in samples containing hornblende. The studied Cpxs fill the compositional gap (Figs. 4 and 5) between Fizh Types I and II lherzolites (Takazawa et al., 2003) and fill the field of the abyssal peridotites. The $\mathrm{Cpxs}(\mathrm{Mg} \#=$ 0.91-0.95) in harzburgites show depleted characteristics with low contents of $\mathrm{Al}_{2} \mathrm{O}_{3}, \mathrm{Na}_{2} \mathrm{O}, \mathrm{Cr}_{2} \mathrm{O}_{3}$, and $\mathrm{TiO}_{2}$ relative to those in lherzolites (Table 1 and Fig. 4). Olivines in lherzolites show low forsterite $(\mathrm{Fo})$ values $(\mathrm{Fo}=89.4-$ 91.5) relative to those of harzburgite (Fo $=90.5-92.5$ ). The composition of olivines is almost the same in both types of lherzolites, i.e., $\mathrm{NiO}=0.3-0.5 \mathrm{wt} \%$ and $\mathrm{MnO}=$ $0.08-0.18 \mathrm{wt} \%$ (Table 1 ). Olivines are similar in chemistry to residual mantle olivines (Takahashi et al., 1987). Orthopyroxenes (Opxs) in lherzolites and harzburgites are enstatite $\left(\operatorname{En}_{85.7-90.9}\right)$ with high $\mathrm{Mg} \#$ values of $0.89-0.92$ and a variable wollastonite content $\left(\mathrm{Wo}_{0.5-5}\right)$ contaminated from Cpx lamellae. Opxs in lherzolites (Type I, II) show wide ranges of $\mathrm{Al}_{2} \mathrm{O}_{3}(2.0-6.7 \mathrm{wt} \%)$ and $\mathrm{CaO}(0.3-2.55$ $w t \%)$ and low concentrations of $\mathrm{Cr}_{2} \mathrm{O}_{3}(0.15-0.92 \mathrm{wt} \%)$ and $\mathrm{TiO}_{2}(0.01-0.14 \mathrm{wt} \%)$ (Table 1). Opxs in harzburgites show a lower composition of $\mathrm{Al}_{2} \mathrm{O}_{3}(2.6 \mathrm{wt} \%$ on average $)$ than that of lherzolites $\left(\mathrm{Al}_{2} \mathrm{O}_{3}=4.7 \mathrm{wt} \%\right.$ on average $)$, but their $\mathrm{Cr}_{2} \mathrm{O}_{3}(0.6 \mathrm{wt} \%$ on average) and $\mathrm{CaO}(0.9 \mathrm{wt} \%$ on average) contents are almost similar to those of lherzolites (Table 1). Primary spinels in basal peridotites exhibit a wide range of $\mathrm{Cr} \#$ values of $0.04-0.53$ and a low $\mathrm{Y}_{\mathrm{Fe}}$ $(<0.05)$ (Table 1and Fig. 6). The spinels are slightly lower in $\mathrm{Cr} \#(0.04-0.20)$ in Type II lherzolites than in Type I $(\mathrm{Cr} \#=0.1-0.25)$, but both spinels have the same $\mathrm{Mg} \#$ (0.68-0.8) and $\mathrm{TiO}_{2}(0.0-0.09$ wt\%) (Figs. 6a and 6c). Spinels in harzburgites are higher in $\mathrm{Cr} \#(0.2-0.53)$ and lower in $\mathrm{Mg} \#(0.55-0.73)$ than those of lherzolites. Hornblendes (Mg\#, 0.88-0.92) show inter-grain heterogeneities in $\mathrm{Al}_{2} \mathrm{O}_{3}(6.0-15.4 \mathrm{wt} \%), \mathrm{Cr}_{2} \mathrm{O}_{3}(0.5-1.4 \mathrm{wt} \%), \mathrm{Na}_{2} \mathrm{O}$ (0.9-3.3 wt\%), and $\mathrm{TiO}_{2}(0.2-1.0 \mathrm{wt} \%)$. 
Table 1. (Continued)

\begin{tabular}{|c|c|c|c|c|c|c|c|c|c|c|c|}
\hline \multirow{3}{*}{$\begin{array}{l}\text { Rock } \\
\text { Sample N. } \\
\text { Minearls }\end{array}$} & \multicolumn{11}{|c|}{ Type II lherzolites } \\
\hline & \multicolumn{5}{|c|}{ Tv.123 } & \multicolumn{4}{|c|}{ G.51 } & \multicolumn{2}{|c|}{ Tv.40 } \\
\hline & Olv & Cpx-c & Cpx-r & Opx & Spl & Olv & $\mathrm{Cpx}$ & Spl & Par.Hb & Cpx-c & Cpx-r \\
\hline $\mathrm{SiO}_{2}$ & 41.19 & 51.68 & 52.64 & 55.01 & 0.00 & 40.73 & 52.13 & 0.00 & 43.50 & 52.46 & 52.40 \\
\hline $\mathrm{TiO}_{2}$ & 0.00 & 0.26 & 0.26 & 0.07 & 0.01 & 0.00 & 0.26 & 0.00 & 0.95 & 0.35 & 0.35 \\
\hline $\mathrm{Al}_{2} \mathrm{O}_{3}$ & 0.00 & 6.82 & 6.07 & 6.01 & 55.78 & 0.00 & 6.85 & 54.48 & 14.77 & 6.80 & 6.37 \\
\hline $\mathrm{Cr}_{2} \mathrm{O}_{3}$ & 0.00 & 0.99 & 0.89 & 0.67 & 12.12 & 0.01 & 1.19 & 12.68 & 0.97 & 0.95 & 0.83 \\
\hline $\mathrm{FeO}^{*}$ & 9.78 & 2.50 & 2.24 & 6.42 & 11.74 & 9.00 & 2.31 & 13.18 & 3.29 & 2.01 & 2.23 \\
\hline $\mathrm{MnO}$ & 0.11 & 0.14 & 0.08 & 0.09 & 0.13 & 0.14 & 0.10 & 0.12 & 0.04 & 0.08 & 0.10 \\
\hline $\mathrm{MgO}$ & 50.16 & 15.07 & 15.32 & 33.03 & 19.05 & 50.76 & 15.34 & 18.99 & 17.19 & 15.36 & 15.44 \\
\hline $\mathrm{CaO}$ & 0.02 & 22.08 & 22.69 & 0.56 & 0.00 & 0.01 & 22.57 & 0.01 & 12.41 & 22.93 & 23.00 \\
\hline $\mathrm{Na}_{2} \mathrm{O}$ & 0.00 & 0.98 & 0.83 & 0.02 & 0.01 & 0.00 & 0.96 & 0.01 & 2.38 & 1.02 & 0.93 \\
\hline $\mathrm{K}_{2} \mathrm{O}$ & 0.00 & 0.00 & 0.00 & 0.00 & 0.00 & 0.00 & 0.00 & 0.00 & 0.00 & 0.00 & 0.00 \\
\hline $\mathrm{NiO}$ & 0.38 & 0.02 & 0.05 & 0.07 & 0.34 & 0.37 & 0.03 & 0.31 & 0.13 & 0.05 & 0.01 \\
\hline Total & 101.6 & 100.5 & 101.1 & 102.0 & 99.2 & 101.0 & 101.7 & 99.8 & 95.6 & 102.0 & 101.7 \\
\hline${ }^{\mathrm{a}} \mathrm{Mg \#}$ & 0.90 & 0.91 & 0.92 & 0.90 & 0.75 & 0.91 & 0.92 & 0.75 & 0.90 & 0.93 & 0.93 \\
\hline${ }^{\mathrm{b}} \mathrm{Fe \#}$ & 0.10 & 0.09 & 0.08 & 0.10 & 0.25 & 0.09 & 0.08 & 0.25 & 0.10 & 0.07 & 0.07 \\
\hline${ }^{\mathrm{c}} \mathrm{Cr} \#$ & & 0.09 & 0.09 & 0.07 & 0.13 & & 0.10 & 0.14 & 0.04 & 0.09 & 0.08 \\
\hline Wo & & 48.96 & 49.52 & 1.09 & & & 49.27 & & & 49.92 & 49.67 \\
\hline En & & 46.48 & 46.53 & 89.06 & & & 46.61 & & & 46.52 & 46.40 \\
\hline $\mathrm{Y}_{\mathrm{Al}}$ & & & & & & & & 0.85 & & & \\
\hline $\mathrm{Y}_{\mathrm{Cr}}$ & & & & & & & & 0.13 & & & \\
\hline $\mathrm{Y}_{\mathrm{Fe} 3+}$ & & & & & & & & 0.02 & & & \\
\hline
\end{tabular}

\begin{tabular}{|c|c|c|c|c|c|c|c|c|}
\hline \multirow{3}{*}{$\frac{\frac{\text { Rock }}{\text { Sample N }}}{\text { Minearls }}$} & \multicolumn{8}{|c|}{ Harzburgites } \\
\hline & \multicolumn{4}{|c|}{ Tv. 60} & \multicolumn{4}{|c|}{ Tv.138 } \\
\hline & Olv & $\mathrm{Cpx}$ & Opx & Spl & Olv & $\mathrm{Cpx}$ & Opx & Spl \\
\hline $\mathrm{SiO}_{2}$ & 42.18 & 53.40 & 57.43 & $\overline{0.00}$ & 41.69 & 52.54 & 57.12 & 0.01 \\
\hline $\mathrm{TiO}_{2}$ & 0.01 & 0.03 & 0.03 & 0.03 & 0.01 & 0.06 & 0.03 & 0.04 \\
\hline $\mathrm{Al}_{2} \mathrm{O}_{3}$ & 0.02 & 2.97 & 2.35 & 36.50 & 0.00 & 3.59 & 2.87 & 41.93 \\
\hline $\mathrm{Cr}_{2} \mathrm{O}_{3}$ & 0.16 & 0.97 & 0.63 & 31.49 & 0.00 & 1.04 & 0.68 & 25.97 \\
\hline $\mathrm{FeO}^{*}$ & 8.44 & 2.19 & 5.79 & 16.73 & 8.41 & 2.23 & 5.53 & 15.01 \\
\hline $\mathrm{MnO}$ & 0.13 & 0.07 & 0.10 & 0.18 & 0.12 & 0.09 & 0.15 & 0.19 \\
\hline $\mathrm{MgO}$ & 51.27 & 17.35 & 34.61 & 14.80 & 51.18 & 17.17 & 34.46 & 16.74 \\
\hline $\mathrm{CaO}$ & 0.11 & 23.76 & 0.85 & 0.05 & 0.00 & 24.06 & 0.92 & 0.01 \\
\hline $\mathrm{Na}_{2} \mathrm{O}$ & 0.00 & 0.02 & 0.00 & 0.00 & 0.00 & 0.01 & 0.00 & 0.00 \\
\hline $\mathrm{K}_{2} \mathrm{O}$ & 0.00 & 0.00 & 0.01 & 0.00 & 0.00 & 0.00 & 0.00 & 0.00 \\
\hline $\mathrm{NiO}$ & 0.35 & 0.05 & 0.08 & 0.15 & 0.40 & 0.07 & 0.08 & 0.16 \\
\hline Total & 102.7 & 100.8 & 101.9 & 99.9 & 101.8 & 100.9 & 101.9 & 100.0 \\
\hline${ }^{\mathrm{a}} \mathrm{Mg \#}$ & 0.92 & 0.18 & 0.91 & 0.64 & 0.92 & 0.16 & 0.92 & 0.70 \\
\hline${ }^{b} \mathrm{Fe \#}$ & 0.08 & 0.07 & 0.09 & 0.36 & 0.08 & 0.07 & 0.08 & 0.30 \\
\hline${ }^{\mathrm{c}} \mathrm{Cr} \#$ & & 0.18 & 0.15 & 0.37 & & 0.16 & 0.14 & 0.29 \\
\hline Wo & & 47.84 & 1.58 & & & 48.35 & 1.73 & \\
\hline En & & 48.60 & 89.84 & & & 48.01 & 89.95 & \\
\hline $\mathrm{Y}_{\mathrm{Al}}$ & & & & 0.62 & & & & 0.69 \\
\hline $\mathrm{Y}_{\mathrm{Cr}}$ & & & & 0.36 & & & & 0.29 \\
\hline $\mathrm{Y}_{\mathrm{Fe} 3+}$ & & & & 0.02 & & & & 0.02 \\
\hline
\end{tabular}

\section{DISCUSSION}

\section{Petrogenesis of basal lherzolites}

Partial melting: origin of Sarami lherzolites. Abyssal peridotites are dominantly spinel lherzolites formed as residues after $\sim 5-15 \%$ melting (e.g., Johnson et al., 1990; Johnson and Dick, 1992; Hellebrand et al., 2001). Based on the spinel composition and Fo content of coexisting olivine (e.g., Arai, 1987, 1994; Pearce et al., 2000) in the basal peridotites (Figs. $6 \mathrm{~b}$ and $6 \mathrm{c}$ ), we infer that Type I and Type II lherzolites are the residues of partial melting with low degrees of melt extraction of less than $12 \%$ (mainly 7\% partial melt) and 10\% (mainly $\sim 5 \%$ ), respectively. However, harzburgites are more depleted relative to lherzolites and were formed by a $10-25 \%$ partial melting of lherzolites (possibly Type I) (Fig. 6). This result is in agreement with the degree of partial melting proposed (on the basis of the spinel $\mathrm{Cr} \#$ ) for a series of abyssal peridotites by Hellebrand et al. (2001). Our preliminary heavy rare earth elements (HREE) analysis of Cpx in basal lherzolites and harzburgites suggests that they experienced a partial melting of less than $10 \%$ for Sarami lherzolites and less than 20\% ( 15\%) for harzburgites (paper in preparation). The linear trends in the $\mathrm{Cpx}$ region of the $\mathrm{Al}_{2} \mathrm{O}_{3}, \mathrm{Na}_{2} \mathrm{O}$, and $\mathrm{TiO}_{2}$ plots (Fig. 4) for Type I lherzolites and harzburgites as well as the trend in the spinel $\mathrm{Cr} \#$ versus $\mathrm{Cpxs} \mathrm{Na}_{2} \mathrm{O}$ plot (Fig. 5) are thought to reflect a residual trend from the Sarami lherzolites to the harzburgites as one single (continuous) series of partial melting. But, Type I and Type II lherzolites (Figs. 4a and 5) show a kinked trend in the $\mathrm{Na}_{2} \mathrm{O}(\mathrm{Cpx})-\mathrm{TiO}_{2}(\mathrm{Cpx})$ plot and the $\mathrm{Na}_{2} \mathrm{O}(\mathrm{Cpx})-\mathrm{Cr} \#$ (spinel) plot, suggesting that 

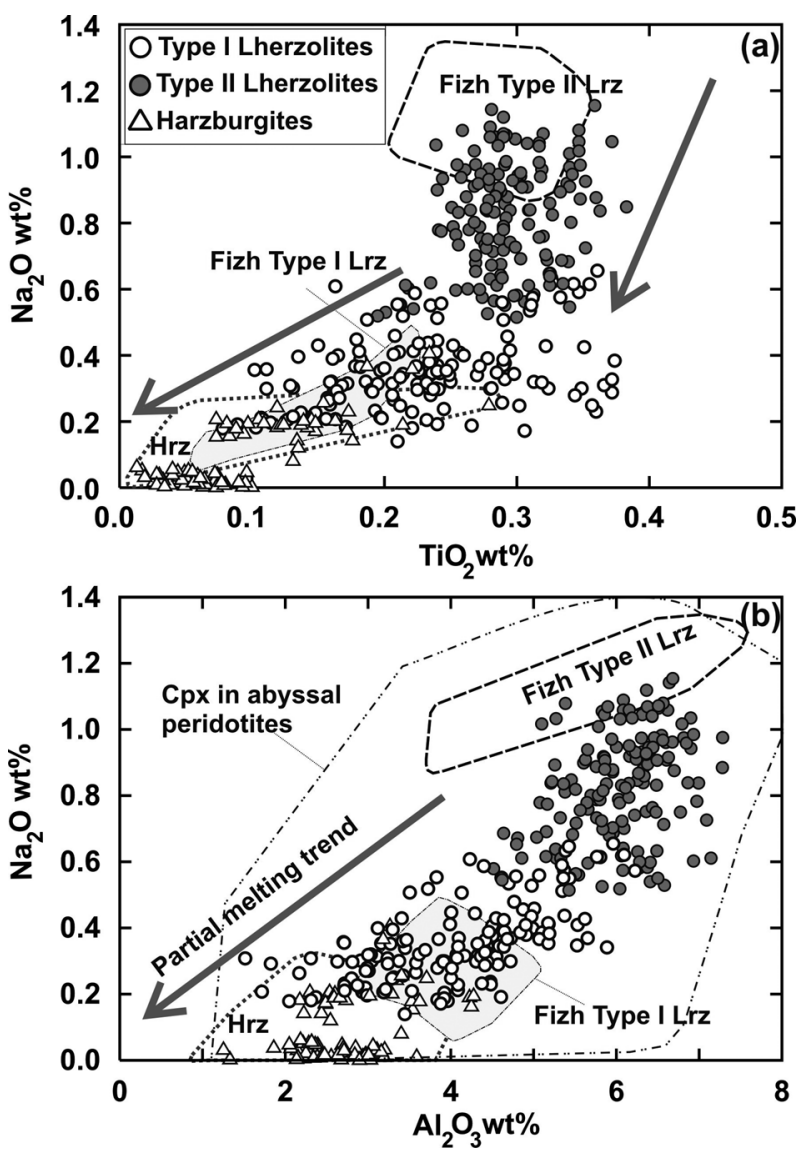

Figure 4. Chemical characteristics of clinopyroxenes in basal peridotites, central Oman ophiolite. (a) $\mathrm{TiO}_{2}$ versus $\mathrm{Na}_{2} \mathrm{O}$ wt\% . (b) $\mathrm{Al}_{2} \mathrm{O}_{3}$ versus $\mathrm{Na}_{2} \mathrm{O}$ wt $\%$. The studied $\mathrm{Cpxs}$ lie in the gap between Fizh Types I (the yellowish-colored field in Fig. 4) and II lherzolites in northern Oman ophiolite (Takazawa et al., 2003), showing good partial-melting trend from fertile lherzolites (Type I) to harzburgites. They are similar in chemistry to Cpxs in abyssal peridotites (e.g., Johnson et al., 1990; Bonatti et al., 1992; Johnson and Dick, 1992).

there are two possible partial melting trends and two separate sources for the Sarami lherzolites. The overlap between Type I and Type II lherzolites in the degree of partial melting (Fig. 6) as well as the high modal volume of Cpx in some Type I samples relative to Type II samples do not favor the derivation of Type I lherzolites from Type II lherzolites via one continuous series of partial melting. Instead, we suggest different sources for Type I and Type II lherzolites. The Na content in Cpx depends on the pressure and degree of partial melting (Blundy et al., 1995; Takazawa et al., 2003); but, the $\mathrm{Al}$ content depends on the degree of partial melting and the equilibrium temperatures (Takazawa et al., 2003; Khedr et al., 2010). The Al and $\mathrm{Na}$ contents decrease from the core to the rim (without a change in the Ti content) of Sarami Cpx porphyroclasts. This decrease is probably due to subsolidus cooling in accordance with low $\mathrm{Al}$ and $\mathrm{Na}$ contents of recrystallized

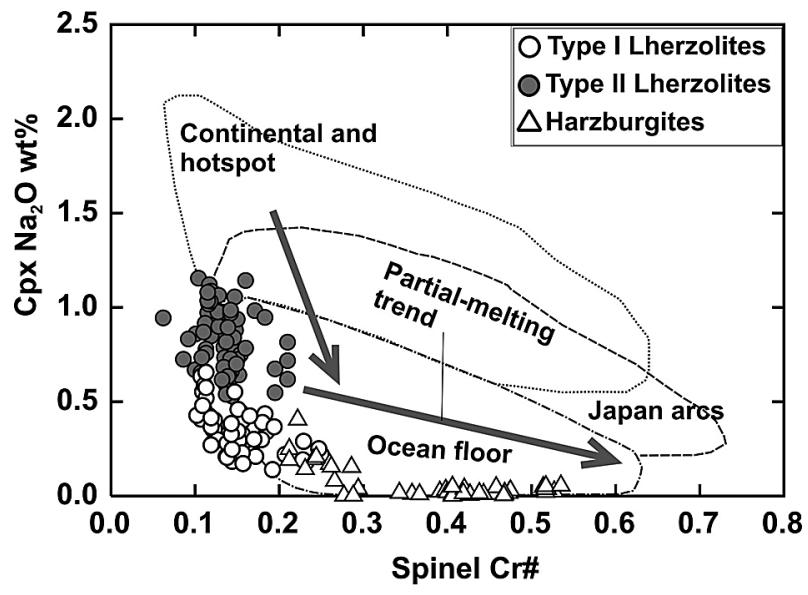

Figure 5. Spinel $\mathrm{Cr} \#$ versus clinopyroxene $\mathrm{Na}_{2} \mathrm{O}$ in basal peridotites. The Cpxs plot of the ocean floor field is from Arai (1991, 1994). Dotted lines that designate the Japan arc, continental rift, hotspots, and ocean floor peridotites are from Arai $(1991,1994)$.

fine Cpx grains associated with $\mathrm{Na}$-bearing hornblende (Table 1) and could have contributed to the kinks in the mineral chemical trends (Figs. 4a and 5). The various degrees of partial melting from lherzolites to harzburgites result in a compositional gradient at the base of the Oman ophiolite, i.e., upper mantle heterogeneity at an oceanic spreading center. The scale of the mantle heterogeneity varies greatly from place to place in the Wadi Sarami area. For example, at Al-Baks, the lithological change from Type II lherzolites at the sole contact to Type I lherzolites and then to harzburgites occurs within a $300 \mathrm{~m}$ (vertical) distance from the sole; whereas at Al-Qala, the transition is more gradational, where harzburgite first occurs at around $400 \mathrm{~m}$ and then grades into dunite at around $1 \mathrm{~km}$ from the sole.

According to the model of Takazawa et al. (2003), the Sarami Type II lherzolites, which are similar in composition to the Fizh Type II lherzolites from north Oman, could have formed via the refertilization of refractory peridotite by freezing of trapped melt, where Sarami Type I lherzolites are of residual origin after melt extraction. Our field observations, mineralogy studies, and chemistry results do not favor this interpretation for the origin of Type II lherzolites. The Sarami Type II lherzolites actually occur as huge homogeneous masses or ridges $(\sim 20-150 \mathrm{~m})$ inside and/or below Type I lherzolites along the contact with the metamorphic sole. It could be difficult for melt refertilization through fractures (e.g., dykes, channels, conduits, etc.) to form such huge homogeneous masses of Type II lherzolites (Fig. 2c). In addition, the basal lherzolites in Wadi Sarami are free of later magmatic activities or melt refertilization through dykes, veins (dunite bands or dunite veins), sills, and melt channels. Therefore, we excluded this method of melt refertilization. However, 

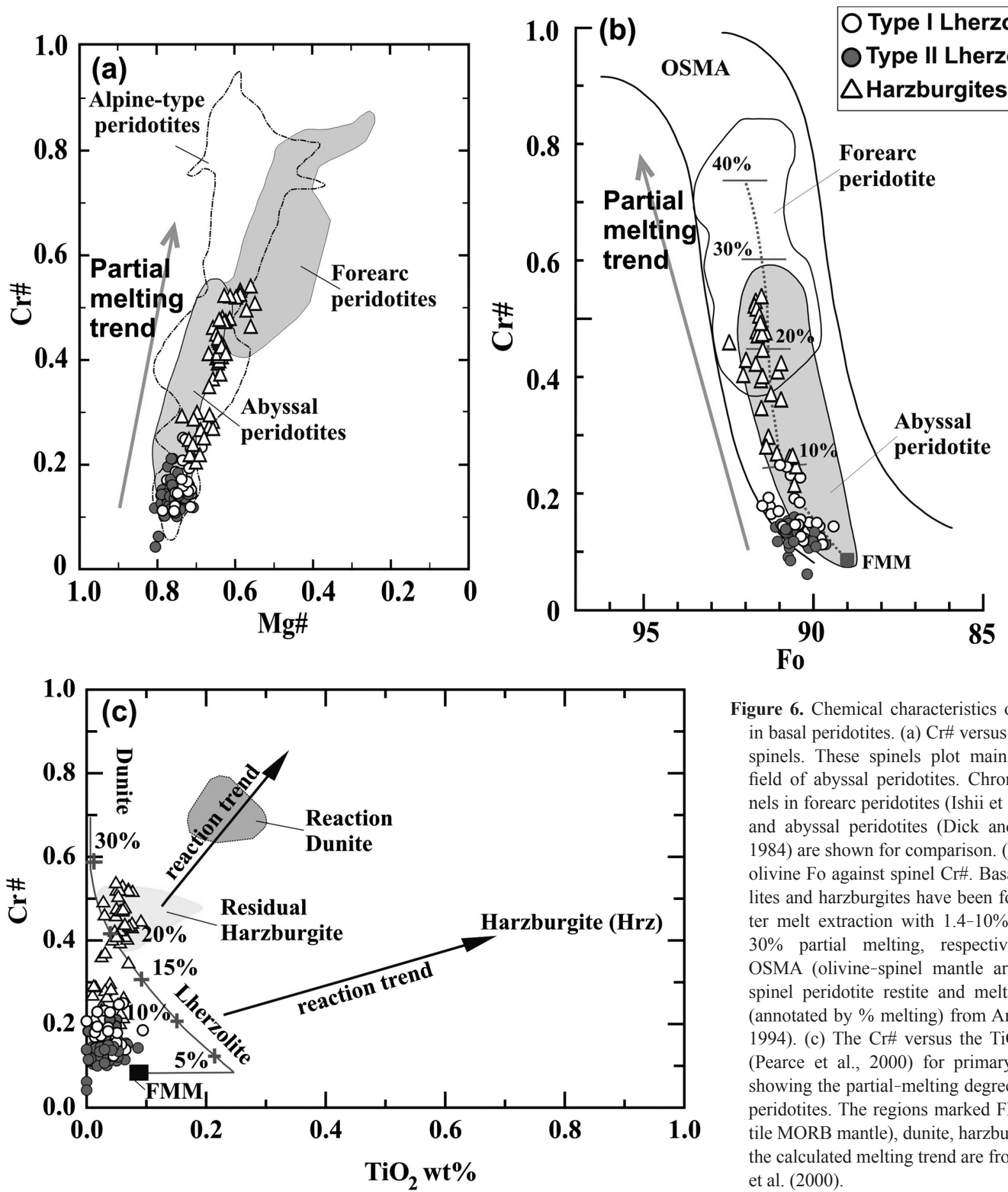

Figure 6. Chemical characteristics of spinels

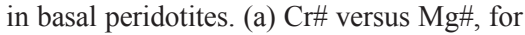
spinels. These spinels plot mainly in the field of abyssal peridotites. Chromian spinels in forearc peridotites (Ishii et al., 1992) and abyssal peridotites (Dick and Bullen, 1984) are shown for comparison. (b) Plot of olivine Fo against spinel Cr\#. Basal lherzolites and harzburgites have been formed after melt extraction with $1.4-10 \%$ and $10-$ $30 \%$ partial melting, respectively. The OSMA (olivine-spinel mantle array) is a spinel peridotite restite and melting trend (annotated by \% melting) from Arai (1987, 1994). (c) The $\mathrm{Cr} \#$ versus the $\mathrm{TiO}_{2}$ model (Pearce et al., 2000) for primary spinels, showing the partial-melting degree of basal peridotites. The regions marked FMM (fertile MORB mantle), dunite, harzburgite, and the calculated melting trend are from Pearce et al. (2000).

refertilization caused by the porous melt flows may form homogenous blocks such as the Sarami Type II lherzolites. But, there is no textural, mineral, and chemical evidence to support such a melt refertilization mechanism. Additionally, our data of $\mathrm{Cpx}$ are continuous, contrary to Cpx in Fizh lherzolites, and we did not observe any gap between the two lherzolite types (Fig. 4). In addition, the high Na-bearing $\mathrm{Cpx}$ and the $\mathrm{Na}$ trend at constant $\mathrm{Ti}$ in Type II lherzolites cannot be explained by LREE-depleted melt refertilization (Fig. 4). Our preliminary trace-element analysis shows that the REE patterns of Cpx in Type I and Type II lherzolites are highly depleted in LREE with a steep slope from middle rare earth elements (MREE) to LREE. This is similar to the residual Cpx after partial melting (Johnson et al., 1990; Johnson and Dick, 1992) and different from the features of Cpx formed and/or modified by melt refertilization. We cannot rule out any metasomatic effect of hydrous fluids in the base of Type 
II lherzolites. The best example of this fluid metasomatism is the formation of replaced hornblende, which has been formed after Cpx during slab (the sole) metasomatism. We recognized Cpx in hydrous peridotites (for a small scale of a few $\mathrm{cm}$ ) at the base of Type II lherzolites, which shows a spoon-shaped REE pattern with high La and Ce values. Such Cpx was possibly formed via a peridotite-sole interaction during ophiolite obduction. In addition, the bulk chemistry of Types I and II lherzolites shows a residual trend similar to that of abyssal peridotites (paper in preparation).

The possible origin of Sarami lherzolites is consistent with a transform-zone model for Fizh basal lherzolites (Takazawa et al., 2003) and Coast-Range ophiolite in California (Choi et al., 2008): the Sarami lherzolites are similar in lithology and chemistry (Cpx, Spl, and Opx) to Coast-Range group-A lherzolites, specifically in spinel $\mathrm{Cr} \#(0.11-0.27)$ and $\mathrm{Cpx}$ composition $(\mathrm{Mg} \#=0.91-0.93$, $\mathrm{Al}_{2} \mathrm{O}_{3}=4-6.0 \mathrm{wt} \%, \mathrm{Na}_{2} \mathrm{O}=0.6-1.1 \mathrm{wt} \%$, and $\mathrm{TiO}_{2}=0.2-$ $0.4 \mathrm{wt} \%$ ) (Choi et al., 2008). Based on the transform-zone model, Type II lherzolites (having $\mathrm{Na}-$ and $\mathrm{Al}$-enriched Cpx) represent a remnant of asthenospheric materials (from a plume or along a large offset transform zone) trapped between the metamorphic lithologies and the base of oceanic lithosphere mantle (Type I) during detachment and obduction. Type I lherzolites are of residual origin and have suffered from high-degree partial melting to form harzburgite at the refractory end (Figs. 4 and 6). The degree of melting progressively decreased toward the failing-ridge (segmentation) system to form fertile lherzolites (e.g., Suhr and Batanova, 1998; Takazawa et al., 2003). For example, Type II lherzolites formed around the paleo-fracture zone that was possibly located to the east of the Sarami block (Nicolas et al., 2000). This is consistent with the model proposed by Takazawa et al. (2003) that explains the occurrence of Fizh basal lherzolites that are close to the inferred failing ridge in the west side of the northern Fizh block (Nicolas et al., 2000). The upper mantle's compositional transition from depleted harzburgites to fertile lherzolites (Type II) toward the paleo-fracture zone has been preserved only in the base of the Sarami block. The vertical gradient of melting degree from lherzolites to harzburgites, which results from a vertical $\mathrm{P}-\mathrm{T}$ change, has been preserved in the Sarami and Wuqbah blocks.

\section{CONCLUSIONS}

This is the first study to document two types of basal lherzolites (Types I and II) from the Wadi Sarami central Oman ophiolite. These two lherzolites were determined based on field occurrences, textures, and mineral compo- sitions. The Type I lherzolites are massive and the Type II ones are foliated. Type II lherzolites crop out only within a direct contact with the sole, and are overlain and/or surrounded by Type I. Their Cpx is enriched in $\mathrm{Al}, \mathrm{Na}, \mathrm{Cr}$, and Ti relative to the Cpx in Type I lherzolites. Type I and II lherzolites are residues of two possible partial melting trends and two separate sources. Lherzolites were formed after low degrees of melt extraction (up to $10 \%$ partial melting) and harzburgites were formed by the partial melting (10-25\%) of lherzolites (possibly Type I). The mineral compositions of Sarami peridotites are similar to those of abyssal peridotites from ridge segments (Figs. 4, $6 \mathrm{a}$ and $6 \mathrm{~b}$ ), suggesting a mid-ocean ridge origin/setting of these peridotites. Type II lherzolites represent a remnant of asthenospheric materials trapped at the base of the oceanic lithosphere mantle (Type I) during detachment and obduction, whereas Type I lherzolites are residuals and form harzburgite after additional partial melting.

\section{ACKNOWLEDGMENTS}

We are grateful to Durair A'Shaikh and Ali Al-Rajhi of the Ministry of Commerce and Industry, Sultanate of Oman, for their help and hospitality during our stay in Oman. We thank Dr. S. Ishimaru, N. Akizawa, H. Negishi, and M. Miura for their assistance during our field expedition to Oman (2010-2012). We thank Drs. T. Mizuka$\mathrm{mi}$ and T. Morishita for their support and discussions in our seminar meeting. We are grateful to reviewers Drs. T. Tsujimori and E. Takazawa for their beneficial comments. We thank Prof. Dr. M. Obata for his fruitful comments and editorial handling of this paper.

\section{REFERENCES}

Arai, S. (1987) An estimation of the least depleted spinel peridotite on the base of olivine-spinel mantle array. Neues Jahrbuch für Mineralogie Monatshefte, 8, 347-354.

Arai, S. (1991) Petrological characteristics of the upper mantle peridotites beneath the Japan island arcs-petrogenesis of spinel peridotites-. Soviet Geology and Geophysics, 32, 8-26.

Arai, S. (1994) Characterization of spinel peridotites by olivinespinel compositional relationships: review and interpretation. Chemical Geology, 113, 191-204.

Blundy, J.D., Falloon, T.J., Wood, B.J. and Dalton, J.A. (1995) Sodium partitioning between clinopyroxene and silicate melts. Journal of Geophysical Research, 100, 15501-15515.

Bonatti, E., Peyve, A., Kepezhinskas, P., Kurentsova, N., Seyler, M., Skolotnev, S. and Udintsev, G. (1992) Upper mantle heterogeneity below the Mid-Atlantic Ridge, $0^{\circ}-15^{\circ} \mathrm{N}$. Journal of Geophysical Research, 97, 4461-4476.

Boudier, F. and Nicolas, A. (1985) Kinematics of oceanic thrusting in the Oman ophiolite: Model of plate convergence. Earth and Planetary Science Letters, 75, 215-222.

Choi, S.H, Mukasa, S.B. and Shervais, J.W. (2008) Initiation of 
Franciscan subduction along a large-offset fracture zone: evidence from mantle peridotites, Stonyford, California. Geology, 36, 595-598.

Dick, H.J.B. and Bullen, T. (1984) Chromian spinel as a petrogenetic indicator in abyssal and alpine-type peridotites and spatially associated lavas. Contributions to Mineralogy and Petrology, 86, 54-76.

Godard, M., Jousselin, D. and Bodinier, J.-L. (2000) Relationships between geochemistry and structure beneath a paleo-spreading centre: A study of the mantle section in the Oman ophiolite. Earth and Planetary Science Letters, 180, 133-148.

Hanghøj, K., Kelemen, P.B., Hassler, D. and Godard, M. (2010) Peridotites from the Wadi Tayin massif, Oman ophiolite; major and trace element geochemistry, and Os isotope and PGE systematics. Journal of Petrology, 51, 201-227.

Hellebrand, E., Snow, J.E., Dick, H.J.B. and Hofmann, A.W. (2001) Coupled major and trace elements as indicators of the extent of melting in mid-ocean-ridge peridotites. Nature, 410, 677-681.

Ishii, T., Robinson, P.T., Maekawa, H. and Fiske, R. (1992) Petrological studies of peridotites from diapiric serpentinite seamounts in the Izu-Ogasawara-Mariana forearc. In Proceedings of the Ocean Drilling Program Scientific Results 125(Fryer, P., Pearce, J.A. and Stokking, L.B. Eds.). Ocean Drilling Program, College Station, TX, 445-485.

Johnson, K.T.M., Dick, H.J.B. and Shimizu, N. (1990) Melting in the oceanic upper mantle; an ion microprobe study of diopsides in abyssal peridotites. Journal of Geophysical Research, 95, 2661-2678.

Johnson, K.T.M. and Dick, H.J.B. (1992) Open system melting and temporal and spatial variation of peridotite and basalt at the Atlantis II fracture zone. Journal of Geophysical Research, 97, 9219-9241.

Khedr, M.Z., Arai, S., Tamura, A. and Morishita, T. (2010) Clinopyroxenes in high-P metaperidotites from Happo-O'ne, central Japan: implications for wedge-transversal chemical change of slab-derived fluids. Lithos, 119, 439-456.

Michibayashi, K., Oohara, T., Satsukawa, T., Ishimaru, S., Arai, S. and Okrugin, V.M. (2009) Rock seismic anisotropy of the low-velocity zone beneath the volcanic front in the mantle wedge. Geophysical Research Letters, 36, L12305, doi: 10.1029/2009GL038527.

Ministry of Petroleum and Minerals (1992) Geological map of Buraymi, scale 1:250,000, Sultanate of Oman, Mascat.

Nicolas, A. (1989) Structures in ophiolites and dynamics of oceanic lithosphere. pp. 367, Kluwer Academy, Norwell, Massachusetts.

Nicolas, A., Boudier, F., Ildefonse, B. and Ball, E. (2000) Accretion of Oman and United Arab Emirates ophiolite-Discussion of a new structural map. Marine Geophysical Researches, 21, 147-179.

Pearce, J.A., Barker, P.F., Edwards, S.J., Parkinson, I.J. and Leat, P.T. (2000) Geochemistry and tectonic significance of peridotites from the south Sandwich arc-basin system, South Atlantic. Contributions to Mineralogy and Petrology, 139, 36-53.

Suhr, G. and Batanova, V. (1998) Basal lherzolites in the Bay of islands ophiolite: origin by detachment-related telescoping of a ridge-parallel melting gradient. Terra Nova, 10, 1-5.

Takahashi, E., Uto, K. and Schilling, J.G. (1987) Primary magma compositions and $\mathrm{Mg} / \mathrm{Fe}$ ratios of their mantle residues along Mid-Atlantic Ridge $29 \mathrm{~N}$ to $73 \mathrm{~N}$. Technical Reports Institute for Study of the Earth's Interior Okayama University Ser A 9, $1-14$.

Takazawa, E., Okayasu, T. and Satoh, K. (2003) Geochemistry and origin of the basal lherzolites from the northern Oman ophiolite (northern Fizh block). Geochemistry Geophysics Geosystems, 227, 1-31.

Manuscript received October 26, 2012

Manuscript accepted December 3, 2012

Published online January 29, 2013

Manuscript handled by Masaaki Obata 